\title{
A Qualitative Study Examining HIV Antiretroviral Adherence Counseling and Support in Community Pharmacies
}

\author{
Jennifer Cocohoba, PharmD, MAS; Megan Comfort, PhD: \\ Hamaseh Kianfar, PhD; and Mallory O. Johnson, PhD
}

\begin{abstract}
BACKGROUND: Observational studies suggest that HIV-focused pharmacies can improve antiretroviral therapy (ART) refill adherence, but there is a lack of clear documentation about the kind and variability of adherence interventions that are conducted.

OBJECTIVE: To use qualitative research methods to obtain an in-depth understanding of how ART adherence support and counseling is provided in human immunodeficiency virus (HIV)-focused community pharmacies. To determine relevant facilitators and barriers around adherence support from both patient's and pharmacist's perspectives.

METHODS: A qualitative research study of patients who patronized and pharmacists who were employed at HIV-focused pharmacies in the San Francisco Bay Area was conducted. Participants were recruited using flyers at HIV clinics and community-based organizations and using blurbs in newsletters. Transcripts were analyzed using grounded theory methods to determine emergent themes in the data.

RESULTS: 19 eligible patients with a self-reported diagnosis of HIV, who were taking their current ART regimen for at least 3 months, and who obtained their ART from a community pharmacy in the San Francisco Bay Area were included; 9 pharmacists who were employed at 9 different pharmacy locations frequented by participants were interviewed. Emergent themes included descriptions of pharmacy adherence counseling and support, roles and responsibilities regarding medication adherence, barriers to providing adherence support, and feeling connected as a facilitator to adherence support relationships.
\end{abstract}

CONCLUSION: Pharmacists provide diverse types of ART adherence support and are uniquely positioned to help clients manage their medications. Additional training on developing relationships with patients and advertising their adherence services may further the role of community pharmacists in supporting antiretroviral adherence.

J Manag Care Pharm. 2013;19(6):454-60

Copyright @ 2013, Academy of Managed Care Pharmacy. All rights reserved.

\section{What is already known about this subject}

- Strict adherence to antiretroviral therapy (ART) at levels 95\% or greater are desirable to keep human immunodeficiency virus (HIV) viral load suppressed to undetectable levels. ${ }^{15-16}$

- Studies have demonstrated that patients using HIV-focused community pharmacies have a $4.9 \%-22.1 \%$ higher mean refill adherence as measured by medication possession ratios or proportion of days covered compared with pharmacies that do not offer specialized HIV services. Typically, ART adherence interventions conducted in these studies are diverse and not well-documented. ${ }^{3-6}$
- Studies of community pharmacists that provide HIV-focused services identify some ART adherence support activities, including in-person or telephone counseling, management of adverse effects, provision of reminder devices and strategies, and facilitation of insurance issues. $5,11,12$

\section{What this study adds}

- This study examined HIV ART adherence support as specifically conducted in HIV-focused community pharmacies. Pharmacists interviewed in this study all reported serving a high volume of HIV-positive patients

- This study elicited HIV patients' experiences and opinions on pharmacy ART adherence support. Examining both the pharmacist's and the patient's desires, needs, and perceptions regarding ART adherence support can give greater direction on changes that might be implemented to improve pharmacy practice.

- This study outlines some considerations for pharmacies that wish to provide HIV-focused services to their clientele such as the need for personalized engagement, training in behavior change techniques to improve adherence counseling, considerations for patient privacy, and linkages to other community-based HIV services.

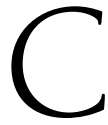

ommunity pharmacists create enhanced pharmacy service models to improve the health of patients with chronic diseases such as human immunodeficiency virus (HIV). These service models are often complex and typically comprise several combined interventions such as medication profile reviews, provision of patient medication records, and adherence support. They are typically assessed using quantitative outcomes; however, for pharmacy interventions that seek to improve medication adherence, qualitative research can add tremendous value. Qualitative research is an exploratory process that attempts to understand perceptions, beliefs, values, and attitudes that underlie an experience such as taking medications or visiting the pharmacy. Although it is more common to the social sciences, there are several applications for pharmacy practice research. Qualitative research can be used to gain an in-depth understanding of patient needs or work-flow processes when developing a clinical service. It can assess the barriers to implementation of a pharmacy service or the parts of a service that are perceived as essential by its users. It may be used prior to the development of a 
quantitative research survey to ensure that all important aspects are included for measurement. Qualitative research typically relies on interview or focus group data, de-emphasizes numerical counts, and often utilizes much smaller samples than are used in quantitative research.

For pharmacies serving patients with HIV, supporting optimal antiretroviral therapy (ART) adherence is essential for patients to maintain health, reduce potential for drug-resistant mutations, and reduce potential for HIV transmission. Recent studies found that patients using pharmacies with HIV-focused services had higher ART refill adherence compared with pharmacies that do not. ${ }^{1-4}$ These studies support pharmacy-based efforts to improve ART adherence. One of the challenges that remains with these large quantitative studies is a lack of clarity as to what comprises the adherence intervention or what the variability in the interventions is across sites. ${ }^{5}$ Our goal in conducting this qualitative research study was to develop a better understanding of the adherence support services that are offered in HIV-specialized pharmacies that serve a highvolume of HIV-positive patients. We hope that this study can provide valuable insight on the adherence support interventions commonly provided in pharmacies as well as patient and provider perceptions of those services. With this information, we will develop a framework for developing ART adherence services in community pharmacies.

\section{Methods}

\section{Recruitment}

HIV-positive subjects were recruited by posting flyers at the San Francisco Women's Interagency HIV Study (WIHS) clinical study site, University of California San Francisco 360 Positive Care Center and Women's HIV Clinics, Catholic Charities Rita da Cascia Family House, and the Tenderloin Resource Center., ${ }^{6,7}$ A recruitment announcement was placed in the biannual WIHS Woman newsletter. Individuals were eligible if they had a self-reported diagnosis of HIV, were taking their current ART regimen for at least 3 months, and obtained ART from a community pharmacy in the San Francisco Bay Area. The criterion for taking ART for greater than 3 months was to ensure that participants had at least 3 recent opportunities to interact with their pharmacy. Patients who never went to a pharmacy and only utilized mail-order or pharmacy delivery services were excluded. Written informed consent was obtained, and subjects were consecutively recruited until saturation occurred $(n=19)$. Saturation is the phenomenon where no new themes emerge despite additional data collection. ${ }^{8}$

To recruit pharmacist study participants, flyers were posted at pharmacies utilized by the enrolled HIV-positive subjects. It became evident that many HIV-positive subjects used 3 specific HIV-focused community pharmacies in San Francisco. To ensure a diverse sample of pharmacists, we expanded recruitment to other HIV-focused pharmacies in the area and allowed pharmacists to contact their colleagues at other HIV-focused pharmacies and ask them to participate. Pharmacists had to be licensed and registered in California, self-report working in a pharmacy serving a high volume of HIV-positive patients, and give verbal consent in order to participate.

\section{Interviews}

A semistructured interview guide tailored to HIV-positive subjects and one tailored to pharmacists were designed for the study. Patient-participants were asked to describe their pharmacy, the staff, and their recent visit. Pharmacists were asked about their pharmacy career, their pharmacy, the patient population they serve, and which patients typically receive adherence support. Both groups were asked to describe their experiences with ART or providing ART adherence support, their opinions on the importance of this support, and barriers to communication around adherence. Questions are further detailed in the Appendix, which is available in the online article. Face-to-face interviews with HIV-positive subjects were performed in a private room by 1 investigator. Interviews took place within a 7-day window of the subject's last pharmacy visit to minimize recall bias and ensure that the subject had a recent pharmacy interaction. If more than 7 days had passed since the last pharmacy visit, the interview was rescheduled. Subjects were offered a $\$ 25$ gift card for their participation. Due to their busy schedules, pharmacists were interviewed via telephone and were offered a $\$ 50$ honorarium for participating. All interviews were digitally audio-recorded and transcribed verbatim by a medical transcription company. The study was approved by the University of California, San Francisco Committee on Human Research.

\section{Analysis}

We used grounded theory methods to develop a conceptual framework for the research. ${ }^{9,10}$ Grounded theory searches for patterns, conducts iterative comparisons, and analyzes outlying cases in order to identify common themes among the data. Our primary interest was to examine the pharmacist-patient dynamic and facilitators and barriers of pharmacy-based ART adherence support. Transcripts were independently reviewed by 2 members of the research team using line-by-line techniques to identify common themes. A preliminary codebook was developed after reading the first 4 interviews; this codebook was refined using axial coding techniques as subsequent transcripts and new elements emerged. Data were imported into ATLAS.ti version 6.2, and the final codebook was applied to all of the data by one researcher.

\section{Results}

\section{Participants}

Nineteen HIV-positive subjects representing 13 different pharmacy locations participated in the semistructured interviews (see Table). All but 2 subjects resided in San Francisco; most had been using their pharmacy for an average of 4 years. Pharmacists $(n=9)$ recruited from 9 different HIV-focused 


\section{TABLE Participant Demographics}

\begin{tabular}{|c|c|c|}
\hline & $\begin{array}{c}\text { Patient- } \\
\text { Participants } \\
(\mathbf{n}=19)\end{array}$ & $\begin{array}{l}\text { Pharmacists } \\
\quad(\mathbf{n}=9)\end{array}$ \\
\hline Male & $11 \%$ & $56 \%$ \\
\hline Age (years), mean ${ }^{\mathrm{a}}$ & 50.8 & - \\
\hline Years practicing as a pharmacist, mean ${ }^{b}$ & - & 10.4 \\
\hline Years going to current pharmacy, mean ${ }^{a}$ & 4.1 & - \\
\hline \multicolumn{3}{|l|}{ Race } \\
\hline White & $32 \%$ & $56 \%$ \\
\hline African American & $68 \%$ & $11 \%$ \\
\hline Other & $0 \%$ & $33 \%$ \\
\hline \multicolumn{3}{|l|}{ Pharmacy type } \\
\hline Retail/chain & $37 \%$ & $33 \%$ \\
\hline Small chain/specialty & $37 \%$ & $33 \%$ \\
\hline Independent & $16 \%$ & $22 \%$ \\
\hline Clinic-associated & $11 \%$ & $11 \%$ \\
\hline
\end{tabular}

pharmacies had been practicing pharmacy for an average of 10 years (range: $3-28$ years).

\section{Emergent Themes Regarding Pharmacy Adherence Counseling and Support}

The primary themes derived from the analyses included (a) descriptions of adherence support provided at HIV-focused pharmacies, (b) roles and responsibility for supporting ART adherence, (c) barriers to providing or accepting adherence support, (d) the feeling of being known by the pharmacy as a facilitator of adherence-supportive relationships, and (e) a need for training in effective adherence communication. Of note, participants and pharmacists often expressed views and experiences that encompassed all medication adherence support, not just specifically for ART.

Adherence Counseling and Support at HIV-Focused Pharmacies. Community pharmacies support ART adherence in multiple ways. Patients who are unable to access their medications due to insurance or other health-system delays are at risk for nonadherence. Patients often highlighted the pharmacist's role in facilitating ART receipt as a very important tangible form of adherence support:

"There's been times where I was waiting for a medication ... and they gave me a couple of pills to hold me until it got okayed, and that's always good to know that your pharmacist will do that for you." (50-year-old white female)

"Sometimes I can say my doctor ordered something for me and my insurance doesn't pay for it, so [the pharmacist] always says that she's going to make sure I get what I need." (43-year-old African American female)

Pharmacists mentioned other tangible forms of adherence support such as enrolling their patients in automatic refill programs, sending patients telephone or text refill reminders, synchronizing medication fill dates to streamline the number of trips that a patient made to the pharmacy to pick up medication, and preparation of reminder packaging such as medicine boxes or bubble packages. Pharmacists were confident that these services had a positive impact on adherence:

"I have patients that can be on 15 drugs per day ... And so we try to get them all together at the same time in a timely manner." (pharmacist, Asian male)

"... call the patient when their refill is due to remind them to come and pick it up, which is something we do, or send it to their house if they're unable or unwilling to come to the pharmacy. If they're not really picking up their medications all that often, I might try to figure out what's going on, if they might want a bubble pack, you know, medi-set type thing to manage their medications a little bit better." (pharmacist, white female)

Pharmacists stated that it was important to counsel patients on maintaining high adherence when dispensing new ART prescriptions. They also described some unsuccessful attempts to counsel poorly adherent patients and expressed mixed feelings regarding their communication effectiveness and doubt regarding patients' openness to discussing antiretroviral adherence:

"For new patients, new consults, and patients who have just been diagnosed or just started their medications ... our initial counseling always involves 'It's really important to take these on a regular basis'. And we kind of go through why it's important, and how you can develop resistance, and then you'll have to change regimens, and your virus is going to gain the upper hand." (pharmacist, white female)

"So, I'll go and talk to patients if I've noticed that they haven't been picking up regularly, and I'll just go and ask them if they've been getting it elsewhere or if they had an extra supply ... Generally, the response is yes, I've had something somewhere. They don't tend to admit that they haven't been taking the medications, to me, at least ... I don't know if there's a better way to approach someone to have them admit that they're not taking them every day or they're having trouble remembering taking them every day or they-yeah, if they are having adverse effects, they're not saying that's the problem." (pharmacist, white female)

"Some of them just don't want to hear you ... Like 'Okay, okay, okay. Yes, well, my doctor told me that already', you know? ... I mean, a lot of them are pretty okay with talking to me. They don't really mind. But a lot of the times, they're not really listening. They're just saying okay." (pharmacist, African American female) 
Roles and Responsibility for Supporting a Patient's ART Adherence. Participants had different opinions on the pharmacist's responsibility to support ART adherence. Patients ascribed higher importance to medication dispensing and generally did not report that supporting adherence was a pharmacist's responsibility. They had difficulty imagining how a pharmacist could have adequate time to support ART adherence because they expected them to spend a majority of their time focusing on dispensing medications correctly:

"I think that a pharmacist has their job cut out for them... I think it's pretty much up to the person to take their medication. I mean, because if the pharmacist went around [calling everyone], he would have a pretty busy job. He'd have a 24/7 job. I mean, he can't be on everybody for their medication ... they're busy, and they got to focus on keeping a clear mind on what they're doing, making sure that the right medicine is getting in the right bottle, going to the right person. Because you make a mistake on somebody's medication, somebody might be allergic to something, and you give them the wrong medication, you can be sued. You can kill somebody." (50-year-old white female)

When patients delineated the team responsible for supporting ART adherence, they placed their own efforts at the center of the model and described a strong sense of personal responsibility to adhere. They expressed the importance of having a trusting partnership with their treating clinician to overcome adherence struggles. Most did not include their pharmacist as an integral member of the adherence team:

Interviewer: "Do you think it's part of the pharmacist's job to help people take their medicines every day?"

Respondent: "No. I think it's up to the people. I think it's up to their provider." (43-year-old African American female)

"It's up to me. It's my job, because I want to live. Yes, I want to make my pharmacist proud of me that he's doing his job well. He's filling my medication well. I can go back and say, 'Fill me up again. Give me another refill.' " (50-year-old white female)

On the contrary, pharmacists reported that detecting nonadherence and providing counseling and support was as important as dispensing the ART correctly. They reported that the adherence services they provided were an essential part of patient care:

" ... a big part of my job is if I'm noticing it's [refilled] late and sitting on my shelf, we're calling them constantly ... Say, if you notice a patient's not being adherent. Keeping in touch with the doctor and letting the doctor know what's going on, too, so that way you can have that 3-way relationship between pharmacy, physician, and patient." (pharmacist, white male)
Barriers to Providing or Accepting Adherence Counseling and Support. Participants described many barriers that deter the provision of adherence support services in the pharmacy setting. Patients expressed frustration regarding complicated insurance systems and anxiety about not being able to receive their medications in a timely manner. Additionally, feeling ill, having concerns about the pharmacy's location, and the presence of drug-seeking or intoxicated pharmacy patrons negatively affected patients' desire to be at the pharmacy and their openness to receiving adherence counseling and support services:

"And the pharmacies, they don't have the medications. They say, 'Can you come back?' And it is like, 'No, I can't come back, I'm sick-that's why I am at the pharmacy!' " (46-yearold white female)

One common barrier to discussing ART adherence with a pharmacist was the lack of privacy in the pharmacy counseling area. None of the patients explicitly mentioned the fear of revealing their HIV-positive status, though pharmacists were sensitive to this concern:

"It's, like, the whole lobby doesn't need to know what I'm getting. Just hand me my stuff ... I'll be trying to whisper ... and [pharmacists will] be all loud and I'm, like, 'Oh, god, please just let me get my medication and get out of here.'" (undisclosed age, African American female)

"They don't want anybody in the pharmacy even knowing what they're taking ... Even if everybody wants to remain confidential, it's very possible that some word could leak out about your HIV status." (pharmacist, white male)

"I do know that at other pharmacies they had this line where you had to wait, you couldn't go beyond a certain point in line. And they don't do that at my pharmacy, and that is one of the things that I don't like about my pharmacy. People are right behind you." (undisclosed age, African American male)

"Some patients, they don't necessarily feel comfortable talking, especially the HIV patients who do come and see me. They don't necessarily want to talk about their HIV medications openly." (pharmacist, Asian male)

Pharmacists may unknowingly erect other adherence communication barriers. Some patients perceived pharmacists as unapproachable and too busy to discuss medications or adherence:

"They don't really talk about too much of anything because they're usually always too busy ... and they don't have a whole lot of people working. So, you've got to try to let them do what they got to do so they don't mess up on their medications." (50-year-old white female)

"I think they have a job to do, and I just think they put up 
a wall, like, they just got to do their part and get it out to the customer, and I don't think they become personable like that." (undisclosed age, African American female)

Pharmacists stated that time stress was a major barrier to discussing medications and adherence with HIV positive patients:

"And given how busy the pharmacy can be, how much time, realistically, on average, can you really spend with a patient just talking to them about their medication? ... when it gets really busy, you might have difficult patients who come in and it might sometimes be hard to spend-especially, if you're the only pharmacist there-to really spend time talking to them." (pharmacist, white male)

Feeling Known by the Pharmacy Facilitates a Relationship that Allows for More In-depth Adherence Support and Counseling. Patients found it valuable and important for their health when they had a more personal relationship with someone working at the pharmacy. This personal relationship was hallmarked by a pharmacy employee recognizing them, acknowledging them, and knowing them by name. Some patients established a connection with a technician or other pharmacy personnel rather than a pharmacist, though patients did not always appear to understand the different roles and responsibilities of pharmacy staff members. The feeling of being known fostered an environment that could facilitate the acceptance of adherence support:

"You can go to some pharmacies and they just say 'here' and send you on your way. I don't know if it's the staff or what it is, but it's like they don't care. When you get a pharmacist who can relate to you, it gives you a different feeling about yourself and the employees at the pharmacy." (59-year-old African American male)

"The guy there, he acknowledges you by your name, and when you come in, he'll say, 'Hi, Miss Johnson' or 'Hi, Miss Jones' or whoever. And 'How you doing today? I got your medicine' or 'I'm sorry ... I'll call you when it's ready' or 'Do you want to wait?' ... He makes you welcome. You're like a family, you're part of that family right there, in that structure." (58-year-old African American female)

Despite their patients' perceptions that they were too busy to talk, most pharmacists recognized the value in establishing a more personal relationship to build trust and open the lines of adherence communication:

"I have about 300-plus HIV patients ... and I know the majority of them by name. And it's very difficult to find that in a community setting." (pharmacist, white male)

"I try to catch people when they're first here at the pharmacy so that they get my name. They know who the pharmacists are. They know that they're free to call if they have questions and we'll help them.... I think that relationship's vital. It's been done away with a little bit in the last decade or two in the pharmacy world, but I do think it's important." (pharmacist, white male)

Most patients could not recall their pharmacist ever speaking with them about ART adherence. In the few instances a patient did recall their pharmacist asking about ART adherence, they responded positively:

"It makes me feel good, it really does. 'Cause that lets me know there is somebody out there besides family, somebody that I really don't know, but just that he's my pharmacist and he shows me and lets me know that he cares." (undisclosed age, African American female)

"Well, they really don't say nothing to me, just 'How you doing, how you feeling? Have you been taking your meds every day?' 'Yeah, I take it.' ... It's very important 'cause that lets me know that they care and that they're not just, 'Here, take your medicine, go on about your business." (46-yearold African American female)

A Need for Training in Effective Adherence Communication. Pharmacists suggested that additional training would improve patient-pharmacist relations, which could, in turn, facilitate the provision of adherence counseling and support. Patients were more concerned that pharmacists were adequately trained to fill medications correctly and that there was adequate staff available to serve the patients, though they did not suggest that additional staff would allow for more one-on-one counseling time or adherence support with a pharmacist:

"If there could be a way that the people who are doing this sort of work-more intensive work dealing with patientscould be somehow better trained or somehow more able to deal with patients, [that would be helpful]." (pharmacist, white male)

"I guess maybe there's a certain kind of skill set of certain things that we would be able to learn to say to them, to drive the point home. Or maybe some kind of a script that would give us a guideline of, okay, here are the things that you want to say to people and what not to say to people." (pharmacist, white female)

\section{Discussion}

Although various quantitative studies support the effectiveness of pharmacies providing ART adherence support, there remains a wide variation in the adherence interventions provided and little guidance on how new pharmacies might begin to offer enhanced HIV services. ${ }^{1-4,5}$ Gaining a baseline understanding of how ART adherence counseling and support is currently provided in U.S. community pharmacies can help 
to guide the development of feasible interventions that can be replicated in other pharmacies. Adherence support techniques described by pharmacists in our San Francisco-based study are similar to those described in another qualitative study: adherence counseling, medication education, telephone reminders to encourage timeliness of prescription refills, and collaboration with treating clinicians. ${ }^{11}$ The barriers to providing ART adherence support reported in our study were similar to those found in 2 qualitative studies, particularly around job time stress and desires for additional training. ${ }^{11,12}$ Our data is consistent with this published literature, and our study also adds valuable insight from the patient's perspective because ART adherence support that is provided by pharmacists may be interpreted differently by their patients.

For pharmacies that are striving to create services for HIVpositive patients, our study highlights several factors that might be considered. Patients in our study appreciated the challenges pharmacists face associated with dispensing, but they otherwise had low expectations with regard to pharmacists providing adherence support. This means that pharmacies may need to invest more time to promote and expound on the personal health benefits of patients engaging in their ART adherence support and counseling services. Our study also suggests that because patients value a personal connection prior to engaging in ART adherence support, blanket approaches to promote adherence services may not be as effective as one-on-one outreach even though large promotional campaigns are certainly more efficient. Patients may be wary of accepting even the best pharmacy services if they are offered by someone with whom they have not established a strong familiarity. Pharmacists can fully engage in treatment advocacy for HIV-positive patients if they are able to create positive expectations and effectively encourage patients to accept adherence services. ${ }^{13}$

Once clients are engaged, pharmacies must consider what adherence support techniques they will employ. Studies suggest that adherence support should be tailored to the individual, and pharmacies must decide on the suite of interventions they will provide, based on their capacity. Pharmacists in our study were more confident providing adherence reminder devices such as bubble packing and refill services such as reminders and home delivery. Pharmacists appeared less confident regarding their effectiveness in adherence counseling. Because patients in our study did not often recall their pharmacists asking about adherence, it appears that pharmacists should increase the frequency with which they assess ART adherence. When pharmacy practices dictate counseling only on new prescriptions, this creates missed opportunities to intervene on adherence at each refill. Forgetting and practical barriers certainly play a role in poor adherence, yet pharmacist counsel- ing efforts should strive to influence more complex adherence issues such as motivation. Additional training in communication, health behavior change, and counseling techniques such as motivational interviewing may increase pharmacist confidence in addressing more challenging adherence cases. ${ }^{14}$

Lastly, our study highlights several additional general opportunities to enhance community pharmacy ART adherence support. Workflow issues such as creating private spaces for counseling and having adequate staff to fulfill dispensing duties may enhance patient engagement in adherence support. Pharmacists are also in a unique position to facilitate referrals, for example, to mental health programs, clinics, substance abuse programs, and other services that can greatly aid ART adherence. Developing relationships with relevant HIV/AIDS community-based organizations and providers can also serve to solidify the pharmacist's role on the adherence health care team. These changes are important steps to shift the role of community pharmacists from medication dispensers to treatment advocates who improve the effectiveness of HIV therapy.

\section{Limitations}

One limitation to our study was that participants were recruited from San Francisco. Opinions and experiences may not be generalizable to other HIV populations and pharmacy practices. We enrolled a limited number of patients and pharmacists, though this was dictated by data saturation. Pharmacists in our study provided care for a high-volume of HIV-infected patients, so although the sample was small, we believe that it captured a reasonable range of ART adherence support techniques that pharmacists might employ. Patients in our study may not have interacted with the pharmacists that we interviewed, but it is likely that they interacted with other pharmacists working at the same locations. Lastly, we did not measure adherence, so it is unclear if our sample was reflective of a highly adherent population that did not need adherence counseling and therefore did not either request or receive it from their pharmacists.

\section{Conclusion}

Pharmacists support ART adherence using a variety of techniques that range from counseling to medication synchronization to use of technology to remind patients to pick up refills. Pharmacies developing HIV specialty services should consider increasing promotion of the benefit of engaging in adherence support, increasing training to improve communication in the pharmacist-patient relationship, and facilitating work spaces that support adherence counseling. These practices and others are important steps to enhance the role of pharmacists as adherence counselors and treatment advocates. 


\section{Authors}

JENNIFER COCOHOBA, PharmD, MAS, is Associate Clinical Professor, Department of Clinical Pharmacy, University of California, San Francisco School of Pharmacy; MEGAN COMFORT, $\mathrm{PhD}$, is Senior Research Sociologist, Urban Health Program, RTI International, San Francisco; HAMASEH KIANFAR, PhD, is Director of Senior Services, Bernal Heights Neighborhood Center, San Francisco; and MALLORY O. JOHNSON, PhD, is Professor, Center for AIDS Prevention Studies, University of California, San Francisco.

AUTHOR CORRESPONDENCE: Jennifer Cocohoba, PharmD, MAS, AAHIVP, Department of Clinical Pharmacy, University of California, San Francisco School of Pharmacy, 521 Parnassus Ave., C-152, Box 0622, San Francisco,

CA 94143-0622. Tel.: 415.514.0892: Fax: 415.476.6632;

E-mail:cocohobaj@pharmacy.ucsf.edu.

\section{DISCLOSURES}

This research was funded by grants from the National Institute of Mental Health, which were obtained by Cocohoba and Johnson. Cocohoba reports that she has also received a grant from Gilead Sciences and travel honoraria unrelated to this research. Comfort reports that she has a grant pending from the National Institutes of Health, is a consultant for The Bridging Group, and has received royalties from the University of Chicago Press and honoraria and travel expenses unrelated to this research. Kianfar reports no conflicts of interest relating to this research. Johnson reports that she has grants pending from NIH, is a member of the Data Safety Monitoring Board for Partners Health, and serves as a consultant for the AIDS Foundation of Chicago, all unrelated to this research.

Study concept and design were contributed primarily by Cocohoba, with assistance from Comfort and input from Johnson and Kianfar. Cocohoba had primary responsibility for data collection, with assistance from Kianfar; data interpretation was the work of Cocohoba, with assistance from Kianfar and input from Comfort and Johnson. The manuscript was written primarily by Cocohoba, with assistance from Comfort and Johnson and was revised primarily by Cocohoba with assistance from Johnson.

\section{REFERENCES}

1. Hirsch JD, Gonzales M, Rosenquist A, Miller TA, Gilmer TP, Best BM. Antiretroviral therapy adherence, medication use, and health care costs during 3 years of a community pharmacy medication therapy management program for Medi-Cal beneficiaries with HIV/AIDS. J Manag Care Pharm. 2011;17(3):213-23. Available at: http://www.amcp.org/WorkArea/ DownloadAsset.aspx?id=8973.
2. Hirsch JD, Rosenquist A, Best BM, Miller TA, Gilmer TP. Evaluation of the first year of a pilot program in community pharmacy: HIV/AIDS medication therapy management for Medi-Cal beneficiaries. J Manag Care Pharm. 2009;15(1):32-41. Available at: http://www.amcp.org/WorkArea/ DownloadAsset. aspx?id=8066.

3. Cocohoba JM, Murphy P, Pietrandoni G, Guglielmo BJ. Improved antiretroviral refill adherence in HIV-focused community pharmacies. J Am Pharm Assoc (2003). 2012;52(5):e67-e73.

4. Murphy P, Cocohoba J, Tang A, Pietrandoni G, Hou J, Guglielmo BJ. Impact of HIV-specialized pharmacies on adherence and persistence with antiretroviral therapy. AIDS Patient Care STDS. 2012;26(9):526-31.

5. Rosenquist A, Best BM, Miller TA, Gilmer TP, Hirsch JD. Medication therapy management services in community pharmacy: a pilot programme in HIV specialty pharmacies. J Eval Clin Pract. 2010;16(6):1142-46.

6. Barkan SE, Melnick SL, Preston-Martin S, et al. The Women's Interagency HIV Study. WIHS Collaborative Study Group. Epidemiology. 1998;9(2):11725

7. Bacon MC, von Wyl V, Alden C, et al. The Women's Interagency HIV Study: an observational cohort brings clinical sciences to the bench. Clin Diagn Lab Immunol. 2005;12(9):1013-19.

8. Kisely S, Kendall E. Critically appraising qualitative research: a guide for clinicians more familiar with quantitative techniques. Australas Psychiatry. 2011;19(4):364-67.

9. Glaser BG, Strauss AL. The Discovery of Grounded Theory: Strategies for Qualitative Research. Chicago: Aldine Publishing Company; 1967.

10. Strauss A, Corbin J. Basics of Qualitative Research. Grounded Theory Procedures and Techniques. Newbury Park, CA: Sage Publishing Company; 1990.

11. Kibicho J, Owczarzak J. Pharmacists' strategies for promoting medication adherence among patients with HIV. J Am Pharm Assoc (2003). 2011;51(6):746-55.

12. Kibicho J, Owczarzak J, Pinkerton SD. Opinions of a small sample of pharmacists about pharmacy setting and patient adherence to antiretroviral therapy. J Manag Care Pharm. 2012;18(6):446-52. Available at: http://www. amcp.org/WorkArea/DownloadAsset.aspx?id=15455.

13. Kibicho J, Owczarzak J. A patient-centered pharmacy services model of HIV patient care in community pharmacy settings: a theoretical and empirical framework. AIDS Patient Care STDS. 2011;26(1):20-28.

14. Hill S, Kavookjian J. Motivational interviewing as a behavioral intervention to increase HAART adherence in patients who are HIV-positive: a systematic review of the literature. AIDS Care. 2012;24(5):583-92.

15. Paterson DL, Swindells S, Mohr J, et al. Adherence to protease inhibitor therapy and outcomes in patients with HIV infection. Ann Intern Med. 2000;133(1):21-30.

16. Nachega JB, Marconi VC, van Zyl GU, et al. HIV treatment adherence, drug resistance, virologic failure: evolving concepts. Infect Disord Drug Targets. 2011;11(2):167-74. 


\section{APPENDIX Semistructured Interview Guide (Online Reference)}

Questions for Patients

1) How long have you lived in the Bay Area?

2) As I explained before, we're interested in learning more about how people use pharmacies. Can you tell me about how you decided to go to the pharmacy that you currently use?

a. Prompts: How long have you been using it? What about this pharmacy made you choose it? How often do you go there?

3) Can you describe the personalities of the pharmacists that work at the pharmacy?

4) What kind of things does the pharmacist talk to you about when discussing your HIV medicines? Can you give some examples of things they might say to you?

a. Prompts: What did you talk about with your pharmacist the last time you went to the pharmacy? How about the time before that? How much time does the pharmacist usually spend talking to you? Would you prefer to spend more, or less, time talking with the pharmacist? How do you usually feel emotionally after talking with the pharmacist?

5) How often does the pharmacist give you information on your HIV medications?

a. Prompts: How important is it to you that the pharmacist talks to you about your HIV medicines?

6) Sometimes pharmacists remind patients to take their medicines every day. Sometimes they try other ways to help patients take their medicines every day. Has your pharmacist ever tried to help you take your HIV medicines regularly?

a. Prompts: What exactly did they say or do for you? Give me an example. Was this helpful? [If yes] Tell me about what was helpful to you. [If no] Tell me about what was not helpful to you.

7) Does your pharmacist think it's important to remind you to take your HIV medicines regularly? What makes you think this?

8) What do you think might help pharmacists talk to their patients? What gets in the way of pharmacists talking to their patients?

a. Prompt: What's the most difficult thing about talking to your pharmacist? How comfortable or uncomfortable are you talking to your pharmacist about missing your medicines? What makes you feel this way?

9) How can pharmacists help patients take their medicines better?

a. Prompt: What would you like to have your pharmacist discuss with you?

10) Most people only see their pharmacist at the pharmacy. How would you feel if your pharmacy called you to see how you were doing with your medicines?

11) How much (if at all) does your pharmacist influence the way you take your medicines?

12) This has been really helpful and interesting. I don't have any more questions for you, but is there anything I left out that you would like to share with me?

\section{Questions for Pharmacists}

1) How long have you been a pharmacist?

a. Prompts: Where did you go to pharmacy school? What made you choose pharmacy as a career? What kinds of jobs have you had as a pharmacist?

2) Tell me a little bit about your pharmacy work environment

a. Prompts: How long have you been working there? How many patients do you serve? Would you describe it as slow, moderate, or busy?

3) Can you describe the patients that come to the pharmacy?

4) How important do you think it is to counsel patients on their medicines?

a. Prompts: How often do you counsel HIV positive patients on their medications? Do your patients feel it's valuable for you to counsel them? How much time do you spend talking to the average patient? Under what circumstances do you spend more time talking to a patient?

5) What kinds of things do you talk about when discussing HIV antiretroviral medicines with a patient?

a. Prompts: Can you give some examples? What if you picked any antiretroviral medication: If I was one of your patients how would you counsel me on this? What is your impression of how patients receive this information from you?

6) When was the last time you counseled a patient on adherence?

a. Prompts: Is this something you do regularly? Why, or why not?

7) How do you (or how would you) counsel a patient on adhering to their antiretroviral medicines?

a. Prompts: What do you say/what would you say? Can you recount some instances when you've counseled a patient on adherence at your pharmacy? What happened?

8) What might help you counsel your patients better?

a. Prompt: What gets in the way of you talking to your patients? What's the most difficult thing about talking to your patients? What's the most difficult thing about talking to your patients about adherence?

9) How do you think pharmacists can help patients take their medicines better?

10) How comfortable would you be calling patients who appear to be missing their medications? Do you think this is a good strategy to assess and help patients with poor adherence? If yes, why? If no, why not, or what would be a good strategy?

11) How much of an impact do you think you have on your patients' medication-taking behavior?

12) This has been very helpful and interesting. These are all the questions I have for you, but is there anything I left out that you would like to share with me? 